\title{
Sedative use of Dexmedetomidine vs. Propofol after Cardiac Surgery: A critical review and meta- analysis.
}

Hesham Abowali

TEAMHealth Research Institute

Matteo Paganini

TEAMHealth Research Institute

Garrett A Enten

TEAMHealth Research Institute

Ayman Elbadawi

University of Texas Medical Branch

Enrico Camporesi ( $\nabla$ enrico_camporesi@teamhealth.com )

Sistema Universitario Ana G Mendez - Campus de South Florida https://orcid.org/0000-0002-65122506

Research article

Keywords: dexmedetomidine; propofol; cardiac surgery; postoperative sedation

Posted Date: October 22nd, 2019

DOI: https://doi.org/10.21203/rs.2.230/v2

License: (c) (1) This work is licensed under a Creative Commons Attribution 4.0 International License. Read Full License 


\section{Abstract}

Abstract Background : The use of dexmedetomidine for sedation post-cardiac surgery is controversial compared to the use of propofol. Methods : A computerized search on Medline, EMBASE, Web of Science, and Agency for Healthcare Research and Quality databases was performed for up to July 2019. Trials evaluating the efficacy of dexmedetomidine versus propofol in the postoperative sedation of cardiac surgery patients were selected. Primary study outcomes were classified as time-dependent (mechanical ventilation time; time to extubation; length of stay in the intensive care unit and the hospital) and nontime dependent (delirium, bradycardia, and hypotension). Results : Our final analysis included 11 RCTs published between 2003 and 2019 and involved a total of 1184 patients. Time to extubation was significantly reduced in the dexmedetomidine group (Standardized Mean Difference (SMD) $=-0.61,95 \%$ Confidence Interval $(\mathrm{Cl})$ : -1.06 to $-0.16, \mathrm{p}=0.008)$, however no difference in mechanical ventilation time was observed (SMD $=-0.72,95 \% \mathrm{Cl}:-1.60$ to 0.15 , N.S.). Moreover, the dexmedetomidine group showed a significant reduction in Intensive Care Unit length of stay ( $S M D=-0.70,95 \% \mathrm{Cl}:-0.98$ to $-0.42, p=0.0005$ ) this did not translate into a reduced hospital length of stay (SMD $=-1.13,95 \% \mathrm{Cl}:-2.43$ to $0.16, N$.S). For non-time dependent factors: incidence of delirium was unaffected between groups (OR: $0.68,95 \% \mathrm{Cl}: 0.43$ to 1.06, N.S.), while the propofol group of patients had higher rates of bradycardia (OR: $3.39,95 \% \mathrm{Cl}: 1.20$ to $9.55, p=0.020$ ) and hypotension (OR: $1.68,95 \% \mathrm{Cl}: 1.09$ to $2.58, p=0.017$ ). Conclusion : Despite the ICU time advantages afforded by dexmedetomidine over propofol, the former does not contribute to an overall reduction in hospital length of stay or an overall improvement in postoperative outcomes for heart valve surgery and CABG patients. Time-dependent outcomes confounded by several factors including variability in staff, site-protocols, and complication rates between individual surgical cases. Keywords: dexmedetomidine; propofol; cardiac surgery; postoperative sedation.

\section{Background}

Cardiac surgery accounts for more than 2 million procedures performed each year worldwide [1-3]. Coronary Arterial Bypass Grafting (CABG) and valvuloplasty represent a large percentage of the cardiac surgeries performed. Despite current advancements in surgical techniques, postoperative complications in cardiac surgery are a frequent event that affect up to $30 \%$ of patients [4-6].

After cardiac surgery, patients must be closely monitored during their stay in the post-anesthesia care unit (PACU) or intensive care unit (ICU) to ensure early detection of acute complications. However, these settings are associated with high incidences of pain, agitation, and delirium. Such stressful conditions can lead to tachycardia, hypertension, immunosuppression, increased oxygen consumption, and increased catecholamine production, potentially contributing to the development of myocardial ischemia $[7,8]$.

Opioids and sedatives are administered to reduce the incidence of postoperative pain, agitation, and delirium in PACU or ICU [7]. In particular, opioids are the gold standard for pain control after cardiac surgery, and titrated sedatives provide the necessary level of hypnosis, amnesia, and anxiolysis. However, 
opioids have numerous side effects - such as nausea, vomiting, urinary retention, decreased gastric motility, pruritus, sedation, and respiratory depression. High dosages of sedatives can lead to prolonged length of stay (LOS) and other drug-specific side effects. Hence, multimodal sedation/analgesia is currently preferred, allowing small doses of several analgesics and sedatives combined to achieve pain and agitation prevention, but at the same time reducing the side effects of any single drug used.

Several studies evaluating the use of dexmedetomidine versus propofol in postoperative sedation of cardiac surgery patients have been previously published, but with limited sample sizes. Propofol is a phenolic derivative, highly lipophilic, intravenous anesthetic agent, with sedative and hypnotic properties; its use after cardiac surgery is well known for its ease of administration, rapid onset, and short awakening time [9]. However, a common side effect of propofol administration is hypotension, and its use as a sedative is limited by the depressant effect on respiration, which can be exacerbated with the administration of opioids $[9,10]$. In contrast, dexmedetomidine is a highly selective alpha-2 adrenergic agonist that provides sympatholytic, sedative, anxiolytic, and analgesic effects without causing respiratory depression [11]. Even if a risk of hypotension and bradycardia is present, its use is growing. Since 2007, dexmedetomidine has been approved for the sedation of non-intubated patients before and during surgical procedures [12].

We have reviewed current literature regarding this subject to define which strategy could improve the quality of sedation and outcomes in post-cardiac surgery patients. Our analysis includes only Randomized Controlled Trials (RCTs) and provides a critical review of the outcomes described in the literature.

\section{Methods}

\section{Search Strategy}

Our study was conducted according to the Preferred Reporting Items for Systematic Reviews and MetaAnalyses (PRISMA) checklist [13] to identify all English language Randomized Controlled Trials (RCTs) that evaluated the use of dexmedetomidine in comparison to propofol for sedation of postoperative cardiac surgery patients. The controlled vocabulary of Medical Subject Headings (MeSH) from PubMed, including subheadings, publication types, and supplementary concepts was used to define the research terms. The search was performed from July 1-10, 2019 on PubMed/Medline, EMBASE, Web of Science, and Agency for Healthcare Research and Quality (AHRQ) using the following selected terms combined with the Boolean operator AND: "dexmedetomidine", "propofol" and "cardiac". Additional post-hoc filters were applied for searches returning more than 300 results. No publication date restrictions were applied. In order to include all possible studies, a further search for grey literature was performed on from July 1013, 2019 (see Supplementary Materials 1 for algorithm details).

Titles and abstracts of identified literature were independently screened by two authors (HA, GE). Literature not complying with the inclusion criteria was excluded. When disagreement occurred, the opinion of a third reviewer (MP) was sought. Records whose full-text was unavailable were excluded in 
the inclusion stage. To further reduce the risk of overlooking pertinent literature, a hand search of reference lists in the included literature and relevant meta-analyses was conducted. The study selection process is described in the flow diagram below [Figure 1].

\section{Inclusion and exclusion criteria}

- Inclusion criteria:

- RCTs;

- use of dexmedetomidine in comparison to propofol for sedation of postoperative surgeries;

- any Cardiac surgery;

$\circ$ age $>18$ years.

- Exclusion criteria:

- non-English literature;

- literature without an available abstract

- literature without available data

\section{Data extraction}

Data extraction was performed by two investigators according to PRISMA guidelines [13] (EA, GA). These basic features were obtained: primary author, publication year, study design and country, along with the number, mean age, and gender of participants [Table 1]. Time to Extubation (TTE), Mechanical Ventilation Time (MVT), Length of Stay in Intensive Care Unit (LOS-ICU), and Length of Stay in the Hospital (LOS-H) were defined as time-dependent outcomes, while other 3 items (delirium, bradycardia, and hypotension) were defined as non-time-dependent [Table 2]. TTE was defined as the number of minutes from intubation in the operating room to extubation, and MVT as the number of minutes that the patient needed mechanical ventilatory support after the arrival in PACU. The two LOS outcomes are referred to as the period of stay in ICU and in the hospital, respectively. These outcomes were selected because they were frequently reported in this field, therefore increasing the external validity of our meta-analysis. For each outcome, the number of studies that addressed the item was specified [Table 2, last row]. Other possible associations depicted by the included studies on other possible outcomes or adverse effects were only discussed if a statistical analysis was considered unreliable.

\section{Risk of Bias}

Two authors (GE, MP) assessed the risk of bias of each included study independently. Disagreements were resolved by consultation with a third author (AE).

Risk of bias was assessed using the following items, according to the Cochrane Collaboration's tool [15, 16]: random sequence generation (selection bias); allocation concealment (selection bias); blinding (performance bias and detection bias), blinding of participants and personnel assessed separately from 
blinding of outcome assessment; incomplete outcome data (attrition bias); selective reporting (reporting bias). Risk of bias was defined as 'low risk', 'high risk' or 'unclear risk' and individual bias items were evaluated as described in the Cochrane Handbook for Systematic Reviews of Interventions [15].

Reporting bias was assessed using funnel plots $[17,18]$; briefly, variables were assessed by plotting standard error (SE) against estimated effect size, SDM for time-dependent variables and log of the odds ratio for non-time dependent variables. The Egger test was utilized as a regression test for funnel plot asymmetry in order to estimate the impact of small study effects. A p-value of $<0.05$ was considered significant.

\section{Data and Quality analysis}

In this manuscript, all statistical analyses were performed using the Comprehensive Meta-Analysis (CMA) software (version 3.0; Biostat Inc., Englewood, NJ, USA). Mean and standard deviation (SD) were used to report continuous variables. Age is only reported as a mean value. When only a median was available, Wan's formula was used to convert median and Inter Quartile Ranges (IQR) into mean and SD values, as it has been demonstrated to be more accurate than Hozo's method for sample sizes exceeding $n=25$ [14]. Categorical variables were described using frequencies. For estimating the effect of each outcome, the Standardized Mean Difference (SMD) and Odds Ratio (OR) are reported at a 95\% Confidence Interval $(\mathrm{Cl})$. The $\mathrm{p}$ values were two-tailed and considered statistically significant if less than 0.05 . Heterogeneity was assessed using Cochran's $Q$ test with $p<0.05$ for statistical significance and $I^{2}$ index $>50 \%$ for substantial heterogeneity. Fixed Effect Model was used if no significant heterogeneity was present. However, if heterogeneity was significant, Random Effect Model was used. In the analysis of the outcomes, six of the outcomes had significant heterogeneity and those included TTE, MVT, LOS-ICU, LOS$\mathrm{H}$, delirium and hypotension.

\section{Results}

Our final analysis included 11 RCTs [Table 1]. These studies were published between 2003 and 2019 and included a total of 1184 patients that underwent heart valve surgery or CABG. Of note, most of the RCTs were carried out in the USA and China (3 studies respectively), and the smallest trial included only 25 patients for each arm [27].

The most assessed outcomes were TTE and LOS-ICU (8/11), followed by delirium (6/11). None of the included trials assessed all the outcomes selected for this meta-analysis at the same time. The study conducted by Liu in 2016 [24] was the only one to assess most of the endpoints (all except MVT) [Table 2]. According to our analysis, TTE was significantly reduced in the dexmedetomidine group (in $7 / 8$ studies, $\mathrm{SMD}=-0.61,95 \% \mathrm{Cl}:-1.06$ to $-0.16, \mathrm{p}=0.008$ ) [Figure 2-A]; however no difference in mechanical ventilation time was observed ( $\mathrm{SMD}=-0.72,95 \% \mathrm{Cl}-1.60$ to 0.15 , N.S.) [Figure 2-B]. Furthermore, dexmedetomidine significantly reduced LOS-ICU (in 8/8 studies; SMD $=-0.70,95 \% \mathrm{Cl}:-0.98$ to -0.42 , $\mathrm{p}=0.0005)$ [Figure 3-A] but this did not translate into a reduced LOS-H (SMD $=-1.13,95 \% \mathrm{Cl}:-2.43$ to 0.16 , 
N.S) [Figure 3-B]. With regard to non-time dependent events, the incidence of delirium was unaffected between groups (OR: $0.68,95 \% \mathrm{Cl}: 0.43$ to 1.06, N.S.) [Figure 4-A]. In contrast, bradycardia (4/4 studies; OR: $3.39,95 \% \mathrm{Cl}: 1.20$ to $9.55, \mathrm{p}=0.020$ ) [Figure 4-B] and hypotension (in 5/5 studies; OR: $1.68,95 \% \mathrm{Cl}$ : 1.09 to $2.58, p=0.017$ ) [Figure $4-C$ ] were significantly more reported with propofol.

As depicted in Table 3, the overall quality of the studies is moderate. Except for one study [28], none of the papers fully met the "blinding of participants and personnel" bias criterion (Table 3). No small-study effects were found as a result of funnel analysis (Supplementary Materials 2).

\section{Discussion}

This work represents a meta-analysis of the largest number of RCTs about the use of dexmedetomidine versus propofol in the sedation following heart valve surgery and CABG currently available, involving 11 RCTs, totaling 1184 patients.

Three main reasons prompted this investigation. First, we wanted to perform a further and more recent meta-analysis comparing dexmedetomidine and propofol, after similar previous publications [1, 30-34], to provide the reader with a more comprehensive statistical analysis of such a comparison. To be more accurate, the comparison was limited RCTs and to those comparing dexmedetomidine and propofol only, in contrast with previous meta-analyses including comparisons between sedatives and placebo [32-35]. Second, studies conducted on an international level, rather than those limited to the United States, were included to provide the reader with a wider perspective, a higher external validity, and conclusions applicable to different healthcare systems. Third, we performed a critical analysis of the endpoints used so far in the literature to emphasize the importance of confounding factors affecting the outcomes on which previous meta-analyses [1, 30-35] were based. In fact, despite the apparent time advantages afforded by dexmedetomidine over propofol, the former does not show particular overall improvements in postoperative care of heart valve surgery and CABG patients.

Time-dependent outcomes assessed in the included studies (TTE, MVT, LOS-ICU, and LOS-H) showed great limitations for two reasons. First, many factors, such as patients' comorbidities, surgical complications, and postoperative bleeding, are well known to influence the postoperative care of cardiac surgery patients [36]. In particular, the Society of Thoracic Surgeons (STS) listed in its database 6 acute postoperative complications of cardiac surgery (re-operation; deep sternal wound infection / mediastinitis; permanent stroke; renal failure; prolonged ventilation [ $>24$ hours]; and new-onset atrial fibrillation) [37], which can happen singularly or, in $4 \%$ of cases, in combination [38]. Each of those is therefore capable of altering the time-dependent endpoints used in the included studies. Second, local protocols and physician's evaluations can affect the decision to extubate the patient, thus changing both TTE and MVT and, accordingly, LOS in ICU and in the hospital.

Regarding TTE, our results are consistent with those of Liu $X$ et al., [1] showing a reduction in TTE in the dexmedetomidine group [Figure 2-A]. On the other hand, MVT seems to be unaffected by the type of sedation in our analysis [Figure 2-B], as previously found by Chang et al. [30]. However, any postoperative 
complication can delay the timing of extubation directly (e.g., prolonged ventilation) or indirectly (e.g., reoperation) and alter the value of these endpoints. Moreover, there is no consensus about the time of extubation after cardiac surgery, and the actual ventilation time that leads to complications is not known [32].

As shown in Figure 3-A and B, dexmedetomidine seems to reduce LOS-ICU but not LOS-H. A prolonged LOS-ICU or LOS-H has several implications, ranging from the increased risk of infections to adverse outcomes and growing financial issues [36]. Anyway, LOS-ICU and LOS-H are affected by the same factors that undermine the reliability of TTE and MVT, and indirectly by all the remaining postoperative complications of cardiac surgery [35]. Since the debate on the best "fast-track cardiac recovery" modality is still ongoing, the possible interference of this lack of consensus with results cannot be ruled out.

Therefore, we think that these time-dependent outcomes are inaccurate in the evaluation of postoperative sedation. In future investigations, it might be possible to stratify patients in classes of postoperative risk of complications and risk of prolonged LOS, and then assess other endpoints, such as the time elapsed between the decision to extubate and the actual extubation time.

Delirium incidence in adult ICU is related to several factors, such as age, mechanical ventilation or pain. Furthermore, delirium is strongly associated with increased ICU mortality and post - ICU cognitive impairment, also establishing a vicious circle with LOS-ICU [7]. Surprisingly, our analysis did not found a difference in the incidence of delirium between the two groups of patients [Figure 4-A]. This is in contrast with previous meta-analyses that found a reduction in delirium incidence with the perioperative use of dexmedetomidine [1, 32-33]. A possible explanation for this result is that our analysis included a more recent trial that favored propofol over dexmedetomidine [29] and could have influenced the results.

Despite decades of experience with propofol in cardiac anesthesia, bradycardia and hypotension are serious concerns that can potentially cause organ hypoperfusion and dysfunction, such as brain damage [9]. Our analysis suggests a significantly diminished incidence of bradycardia and hypotension in dexmedetomidine sedated patients following heart valve surgery and CABG. However, such adverse effects have also been reported with the use of dexmedetomidine, even if promptly resolved with fluid boluses. This suggests the need for close advanced monitoring with at least heart rhythm and noninvasive blood pressure regardless of the agent used for sedation.

Apart from the critic about time-dependent endpoints, several limitations were noted during our analysis. Firstly, analgesic therapy used to reduce pain during post - surgery (mainly opioids) showed high variability in dosage and different starting and ending points of administration that were not clearly stated. This variability was noted also with regard to other sedatives (e.g., benzodiazepines) used during the surgeries, without a standardized protocol for general anesthesia. Such variability could have affected both time-dependent outcomes and especially delirium, which has a number of influencing factors precipitating this clinical condition. Future trials should take into account these aspects and try to follow a standardized protocol for the administration of intraoperative general anesthesia and of postoperative analgesics. 
Although our study intended to include all types of cardiac surgery, the search did not return any paper regarding cardiac transplants in the adult population. The post-cardiac transplant sedation has been covered only on the pediatric population, in which dexmedetomidine showed better prevention of opioid withdrawal syndrome [39]. However, more efforts are needed to evaluate sedation after this important subset of cardiac surgeries, with particular attention to the sympatholytic effects of dexmedetomidine on a denervated transplanted heart.

Additionally, the sedation scales used in these studies (RASS and Ramsey scores) are often affected by incorrect evaluation by healthcare professionals [40], and are validated on sedatives in general but not specifically on dexmedetomidine (that has a specific alpha -2 receptor agonism related sedation mechanism). Since the sedation of dexmedetomidine resembles natural sleep [42], future studies should closely assess this particular aspect of sedation level evaluation.

Finally, the medical authorities globally, except for 2 countries, recommend limiting the use of dexmedetomidine to 24 hours. This may limit the benefits seen from using such a sedative in the postoperative setting of cardiac surgery patients [41].

\section{Conclusions}

This meta-analysis did not find particular advantages in the use of dexmedetomidine compared to propofol for the sedation of post-cardiac surgery patients. Only bradycardia and hypotension showed a significant reduction in patients sedated with dexmedetomidine, while the results on the other endpoints are questionable. Time-dependent parameters, widely used in previous trials in this field, are inaccurate due to several reasons and are not reliable for a proper evaluation of the benefits of dexmedetomidine versus propofol.

More efforts are needed to find new reliable outcomes for this evaluation, to standardize sedation protocols in post-cardiac surgery patients, and to assess mid- and long-term outcomes in the two groups.

\section{Abbreviations}




\begin{tabular}{ll} 
CVDs & Cardiovascular Diseases \\
\hline CABG & Coronary Arterial Bypass Grafting \\
\hline Cl & Confidence Interval \\
\hline CMA & Comprehensive Meta-Analysis \\
\hline ICU & Intensive Care Unit \\
\hline IQR & Inter Quartile Ranges \\
\hline LOS & Length of Stay \\
\hline LOS-H & Length of Stay in the Hospital \\
\hline LOS-ICU & Length of Stay in Intensive Care Unit \\
\hline MeSH & Medical Subject Headings \\
\hline MVT & Mechanical Ventilation Time \\
\hline OR & Odds Ratio \\
\hline PACU & Post-anesthesia Care Unit \\
\hline PRISMA & Preferred Reporting Items for Systematic Reviews and Meta-Analyses \\
\hline SD & Standard Deviation \\
\hline SMD & Standardized Mean Difference \\
\hline TTE & Time to Extubation \\
\hline
\end{tabular}

\section{Declarations}

- Ethics approval and consent to participate

- Not applicable as manuscript is a meta-analysis of existing literature

- Consent for publication

- All listed authors agree with the submission of "Sedative use of Dexmedetomidine vs. Propofol after Cardiac Surgery: A critical review and meta-analysis" to BMC Anesthesiology

- Availability of data and material

- All data generated or analysed during this study are included in this published article. For supplementary data please refer to the list of references in the works cited, restrictions may apply to the availability of supplementary data based on third party licensing.

- Competing interests 
- Authors declare no competing interests

- Funding

- No outside funding was used in support of this manuscript. This manuscript is a non-sponsored literature review.

- Authors' contributions

- Hesham A. Abowali

- Data Analysis

- Primary Author

- Matteo Paganini

- Data Analysis

- Secondary Author

- Ayman Elbadawi

- Experimental Design

- Data Analysis

- Tertiary Editor

- Garrett Enten

- Experimental Design

- Secondary Editor

- Tertiary Author

- Enrico M. Camporesi

- Experimental Design

- Primary Editor

- Quaternary Author

- Acknowledgements

- None

\section{References}

1. Liu X, Xie G, Zhang K, Song S, Song F, Jin Y, et al. Dexmedetomidine vs propofol sedation reduces delirium in patients after cardiac surgery: A meta-analysis with trial sequential analysis of randomized controlled trials. J Crit Care. 2017;38:190-196. 2. World health statistics 2018: monitoring health for the SDGs, sustainable development goals. Geneva: World Health Organization; 2018. Available at: 
http://apps.who.int/iris/bitstream/handle/10665/272596/9789241565585-eng?ua=1. Accessed September 10, 2018. 3. Global Atlas on Cardiovascular Disease Prevention and Control. Mendis S, Puska P, Norrving B editors. World Health Organization, Geneva 2011. Available at:

http://http://www.who.int/cardiovascular_diseases/publications/atlas_cvd/en/ . Accessed September 10, 2018. 4. Shahian DM, O'Brien SM, Filardo G, Ferraris VA, Haan CK, Rich JB, et al. The Society of Thoracic Surgeons 2008 cardiac surgery risk models: part 1-coronary artery bypass grafting surgery. Ann Thorac Surg. 2009;88(1 Suppl):S2-22. 5. O'Brien SM, Shahian DM, Filardo G, Ferraris VA, Haan CK, Rich JB, et al. The Society of Thoracic Surgeons 2008 cardiac surgery risk models: part 2-isolated valve surgery. Ann Thorac Surg. 2009;88(1 Suppl):S23-42. 6. Shahian DM, O'brien SM, Filardo G, Ferraris VA, Haan CK, Rich JB, et al. The Society of Thoracic Surgeons 2008 cardiac surgery risk models: part 3-valve plus coronary artery bypass grafting surgery. Ann Thorac Surg. 2009;88(1 Suppl):S43-62. 7. Barr J, Fraser GL, Puntillo K, Ely EW, Gélinas C, Dasta JF, et al. Clinical practice guidelines for the management of pain, agitation, and delirium in adult patients in the intensive care unit. Crit Care Med. 2013;41(1):263-306. 8. Epstein J, Breslow MJ. The stress response of critical illness. Crit Care Clin. 1999;15(1):17-33 9. Mckeage K, Perry CM. Propofol: a review of its use in intensive care sedation of adults. CNS Drugs. 2003;17(4):235-72. 10. Barr J, Donner A. Optimal intravenous dosing strategies for sedatives and analgesics in the intensive care unit. Crit Care Clin. 1995;11(4):827-47. 11. Precedex [package insert]. North Chicago,IL:Abbott laboratories; 2001). 12. Gerlach AT, Murphy CV, Dasta JF. An updated focused review of dexmedetomidine in adults. Ann Pharmacother. 2009;43(12):2064-74. 13. Moher D, Liberati A, Tetzlaff J, Altman DG. Preferred reporting items for systematic reviews and meta-analyses: the PRISMA statement. PLoS Med. 2009;6(7):e1000097. 14. Wan X, Wang W, Liu J, Tong T. Estimating the sample mean and standard deviation from the sample size, median, range and/or interquartile range. BMC Med Res Methodol. 2014;14:135. 15. Higgins JPT, Green S (editors). Cochrane Handbook for Systematic Reviews of Interventions Version 5.1.0 [updated March 2011]. The Cochrane Collaboration, 2011. Available from www.cochrane-handbook.org. 16. Higgins JPT, Altman DG, Gøtzsche PC, Jüni P, Moher D, Oxman AD, et al. The Cochrane Collaboration's tool for assessing risk of bias in randomised trials. BMJ 2011;343: d5928. 17. Baujat, B., Mahe, C., Pignon, J.-P., \& Hill, C. (2002). A graphical method for exploring heterogeneity in meta-analyses: Application to a meta-analysis of 65 trials. Statistics in Medicine, 21(18), 2641-2652. 18. Olkin, I., Dahabreh, I. J., \& Trikalinos, T. A. (2012). GOSH - a graphical display of study heterogeneity. Research Synthesis Methods, 3, 214-223. 19. Herr DL, Sum-ping ST, England M. ICU sedation after coronary artery bypass graft surgery: dexmedetomidine-based versus propofol-based sedation regimens. J Cardiothorac Vasc Anesth. 2003;17(5):576-84. 20. Corbett SM, Rebuck JA, Greene CM, Callas PW, Neale BW, Healey MA, et al. Dexmedetomidine does not improve patient satisfaction when compared with propofol during mechanical ventilation. Crit Care Med. 2005 May;33(5):940-5. 21. Maldonado JR, Wysong A, Van der starre PJ, Block T, Miller C, Reitz BA. Dexmedetomidine and the reduction of postoperative delirium after cardiac surgery. Psychosomatics. 2009;50(3):206-17. 22. Karaman Y, Abud B, Tekgul ZT, Cakmak M, Yildiz M, Gonullu M. Effects of dexmedetomidine and propofol on sedation in patients after coronary artery bypass graft surgery in a fast-track recovery room setting. $J$ Anesth. 2015;29(4):522-8. 23. Djaiani G, Silverton N, Fedorko L, et al. Dexmedetomidine versus Propofol Sedation Reduces Delirium after Cardiac Surgery: A Randomized Controlled Trial. Anesthesiology. 
2016;124(2):362-8. 24. Liu X, Zhang K, Wang W, Xie G, Fang X. Dexmedetomidine sedation reduces atrial fibrillation after cardiac surgery compared to propofol: a randomized controlled trial. Crit Care. 2016;20(1):298. 25. Liu X, Zhang K, Wang W, Xie G, Cheng B, Wang Y, et al. Dexmedetomidine Versus Propofol Sedation Improves Sublingual Microcirculation After Cardiac Surgery: A Randomized Controlled Trial. J Cardiothorac Vasc Anesth. 2016 Dec;30(6):1509-1515. 26. Mogahd MM, Mahran MS, Elbaradi GF. Safety and efficacy of ketamine-dexmedetomidine versus ketamine-propofol combinations for sedation in patients after coronary artery bypass graft surgery. Ann Card Anaesth. 2017;20(2):182-187. 27. Elgebaly AS, Sabry M. Sedation effects by dexmedetomidine versus propofol in decreasing duration of mechanical ventilation after open heart surgery. Ann Card Anaesth. 2018;21(3):235-242. doi:10.4103/aca.ACA_168_17 28. Sheikh TA, Dar BA, Akhter N, Ahmad N. A Comparative Study Evaluating Effects of Intravenous Sedation by Dexmedetomidine and Propofol on Patient Hemodynamics and Postoperative Outcomes in Cardiac Surgery. Anesth Essays Res. 2018;12(2):555-560. doi:10.4103/aer.AER_46_18 29. Shi C, Jin J, Qiao L, Li T, Ma J, Ma Z. Effect of perioperative administration of dexmedetomidine on delirium after cardiac surgery in elderly patients: a double-blinded, multi-center, randomized study. Clin Interv Aging. 2019;14:571-575. Published 2019 Mar 15. doi:10.2147/CIA.S194476 30. Chang L, Fang H, Xie H, Wang C, Zhu J, Li M, et al. Evaluation of dexmedetomidine versus propofol sedation therapy in mechanically ventilated cardiac surgery patients: A systematic review and meta-analysis of randomized controlled trials and observational studies. International Journal of Clinical and Experimental Medicine, 2016;9(6):9694- 9704. 31. Tan JA, Ho KM. Use of dexmedetomidine as a sedative and analgesic agent in critically ill adult patients: a meta-analysis. Intensive Care Med. 2010;36(6):926-39. 32. Geng J, Qian J, Cheng H, Ji F, Liu H. The Influence of Perioperative Dexmedetomidine on Patients Undergoing Cardiac Surgery: A Meta-Analysis. PLoS ONE. 2016;11(4):e0152829. 33. Wu M, Liang Y, Dai Z, Wang S. Perioperative dexmedetomidine reduces delirium after cardiac surgery: A meta-analysis of randomized controlled trials. J Clin Anesth. 2018 Nov;50:33-41. 34. Nguyen J, Nacpil N. Effectiveness of dexmedetomidine versus propofol on extubation times, length of stay and mortality rates in adult cardiac surgery patients: a systematic review and metaanalysis. JBI Database System Rev Implement Rep. 2018 May;16(5):1220-1239. 35. Goeddel LA, Hollander KN, Evans AS. Early Extubation After Cardiac Surgery: A Better Predictor of Outcome than Metric of Quality?. J Cardiothorac Vasc Anesth. 2018;32(2):745-747 36. D'agostino RS, Jacobs JP, Badhwar V, et al. The Society of Thoracic Surgeons Adult Cardiac Surgery Database: 2019 Update on Outcomes and Quality. Ann Thorac Surg. 2018. 37. Crawford TC, Magruder JT, Grimm JC, et al. Complications After Cardiac Operations: All Are Not Created Equal. Ann Thorac Surg. 2017;103(1):32-40. 38. Doering L, Esmailian F, Lacks H: Perioperative predictors of ICU and hospital costs in coronary artery bypass graft surgery. Chest 2000;118:736-743. 39. Vega L, Sanchez-de-toledo J, Gran F, Ortega J, Pujol M, Balcells J. Prevention of opioid withdrawal syndrome after pediatric heart transplantation: usefulness of dexmedetomidine. Rev Esp Cardiol (Engl Ed). 2013;66(7):593-5. 40. Riessen R, Pech R, Tränkle P, Blumenstock G, Haap M. Comparison of the Ramsay score and the Richmond agitation sedation score for the measurement of sedation depth. Critical Care. 2012;16(Suppl 1):P326. 41. Yu S-B.

Dexmedetomidine sedation in ICU. Korean Journal of Anesthesiology. 2012;62(5):405-411. 42. Kunisawa T. Dexmedetomidine hydrochloride as a long-term sedative. Ther Clin Risk Manag. 2011;7:291-9. 


\section{Tables}

Table 1: baseline characteristics of the 11 included trials.

\begin{tabular}{|l|l|l|l|l|l|}
\hline $\begin{array}{l}\text { Primary } \\
\text { Author }\end{array}$ & $\begin{array}{l}\text { Publication } \\
\text { Year }\end{array}$ & $\begin{array}{l}\text { Type of Study/ } \\
\text { Country of Origin }\end{array}$ & $\begin{array}{l}\text { No of patients } \\
\text { (Dex/ Propofol) }\end{array}$ & $\begin{array}{l}\text { Mean } \\
\text { Age } \\
\text { (Dex } \\
\text { Propofol) }\end{array}$ & $\begin{array}{l}\text { \%Male } \\
\text { (Dex/ } \\
\text { Propofol }\end{array}$ \\
\hline Herr & 2003 & RCT/USA & $148 / 147$ & $61.9 / 62.4$ & $93 / 87$ \\
\hline Corbett & 2005 & RCT/USA & $43 / 46$ & $63 / 61$ & $76 / 76$ \\
\hline Maldonado & 2009 & RCT/USA & $30 / 30$ & $55 / 58$ & $65 / 58$ \\
\hline Karaman & 2015 & RCT/Turkey & $31 / 33$ & $62.5 / 63.9$ & $83.8 / 87.9$ \\
\hline Djaiani & 2016 & RCT/ Canada & $91 / 92$ & $72.7 / 72.4$ & $74.7 / 76$ \\
\hline Liu a & 2016 & RCT/China & $44 / 44$ & $53 / 56.5$ & $47.7 / 31.8$ \\
\hline Liu b & 2016 & RCT/China & $29 / 32$ & $53 / 55$ & $34 / 47$ \\
\hline Mogahd & 2017 & RCT/Egypt & $35 / 35$ & $53.5 / 54.9$ & $51.4 / 57$ \\
\hline Elgebaly & 2018 & RCT/Egypt & $25 / 25$ & $53.7 / 52.5$ & $50 / 30$ \\
\hline Sheikh & 2018 & RCT/India & $30 / 30$ & $33.6 / 35.6$ & $60 / 40$ \\
\hline Shi & 2019 & RCT/ China & $84 / 80$ & $74.7 / 74.4$ & $75 / 70$ \\
\hline P-value & & & & N.S & N.S \\
\hline
\end{tabular}

RCT: Randomized Controlled Trial; Dex: dexmedetomidine.

Table 2: Outcomes assessed in the 11 included trials. 


\begin{tabular}{|c|c|c|c|c|c|c|c|}
\hline \multirow{2}{*}{$\begin{array}{c}\text { Study } \\
\text { Study (year) }\end{array}$} & \multicolumn{4}{|c|}{ Time dependent } & \multicolumn{3}{|c|}{ Non-time dependent } \\
\hline & TTE & MVT & LOS ICU & LOS-H & Delirium & Bradycardia & Hypotension \\
\hline $\begin{array}{l}\text { Herr [15] } \\
\text { (2003) }\end{array}$ & $\mathrm{Y}$ & $Y$ & $\mathrm{~N}$ & $\mathrm{~N}$ & $\mathrm{~N}$ & $\mathrm{Y}$ & $\mathrm{Y}$ \\
\hline $\begin{array}{l}\text { Corbett [16] } \\
\text { (2005) }\end{array}$ & $\mathrm{N}$ & $Y$ & $Y$ & $\mathrm{~N}$ & $\mathrm{~N}$ & $\mathrm{~N}$ & $\mathrm{Y}$ \\
\hline $\begin{array}{c}\text { Maldonado [18] } \\
\text { (2009) }\end{array}$ & $Y$ & $\mathrm{~N}$ & $Y$ & $Y$ & $Y$ & $\mathrm{~N}$ & $\mathrm{~N}$ \\
\hline $\begin{array}{c}\text { Karaman [24] } \\
\text { (2015) }\end{array}$ & $Y$ & $\mathrm{~N}$ & $\mathrm{~N}$ & $\mathrm{~N}$ & $\mathrm{~N}$ & $Y$ & $Y$ \\
\hline $\begin{array}{c}\text { Djaiani [25] } \\
\text { (2016) }\end{array}$ & $Y$ & $\mathrm{~N}$ & $Y$ & $Y$ & $Y$ & $\mathrm{~N}$ & $\mathrm{~N}$ \\
\hline $\begin{array}{l}\text { Liu a [26] } \\
\text { (2016) }\end{array}$ & $Y$ & $\mathrm{~N}$ & $Y$ & $Y$ & $Y$ & $Y$ & $Y$ \\
\hline $\begin{array}{l}\text { Liu b [27] } \\
\text { (2016) }\end{array}$ & $Y$ & $\mathrm{~N}$ & $\mathrm{~N}$ & $\mathrm{~N}$ & $Y$ & $Y$ & $Y$ \\
\hline $\begin{array}{c}\text { Mogahd [28] } \\
\text { (2017) }\end{array}$ & $\mathrm{Y}$ & Y & Y & $\mathrm{N}$ & $\mathrm{N}$ & $\mathrm{N}$ & $\mathrm{N}$ \\
\hline $\begin{array}{c}\text { Elgebaly [44] } \\
\text { (2018) }\end{array}$ & $\mathrm{N}$ & Y & Y & $\mathrm{N}$ & $\mathrm{N}$ & $\mathrm{N}$ & $\mathrm{N}$ \\
\hline $\begin{array}{c}\text { Sheikh [45] } \\
\text { (2018) }\end{array}$ & $\mathrm{N}$ & $Y$ & $Y$ & $\mathrm{~N}$ & $Y$ & $\mathrm{~N}$ & $\mathrm{~N}$ \\
\hline
\end{tabular}


Y

Y

$\mathrm{N}$

$\mathrm{N}$

(2019)

No. of studies addressing the outcome
\begin{tabular}{l} 
(out of 11) \\
\hline
\end{tabular}

TTE: Time To Extubation; MVT: Mechanical Ventilation Time; LOS-ICU: Length Of Stay in Intensive Care Unit; LOS-Hosp: Length Of Stay in the hospital; Y: yes (assessed); N: no (not assessed). TTE and LOS-ICU were the most assessed outcomes. None of the studies assessed all the outcomes at the same time. The study conducted by Liu in 2016 [26] was the only to assess most of the endpoints (all except MVT).

Table 3: Risk of Bias in the 11 included trials. 


\begin{tabular}{|c|c|c|c|c|c|c|c|}
\hline $\begin{array}{c}\text { Author / } \\
\text { date }\end{array}$ & $\begin{array}{l}\text { Random } \\
\text { sequence } \\
\text { generation }\end{array}$ & $\begin{array}{c}\text { Allocation } \\
\text { concealment }\end{array}$ & $\begin{array}{l}\text { Blinding of } \\
\text { participants } \\
\text { and } \\
\text { personnel }\end{array}$ & $\begin{array}{l}\text { Blinding of } \\
\text { outcome } \\
\text { assessment }\end{array}$ & $\begin{array}{l}\text { Incomplete } \\
\text { outcome } \\
\text { data }\end{array}$ & $\begin{array}{l}\text { Selective } \\
\text { reporting }\end{array}$ & $\begin{array}{l}\text { Other } \\
\text { sources } \\
\text { of bias }\end{array}$ \\
\hline Herr (2003) & Uncertain & Low Risk & High Risk & Uncertain & Low Risk & Uncertain & $\begin{array}{l}\text { Low } \\
\text { Risk }\end{array}$ \\
\hline $\begin{array}{l}\text { Corbett } \\
(2005)\end{array}$ & Low Risk & Low Risk & High Risk & Uncertain & Low Risk & Uncertain & $\begin{array}{l}\text { Low } \\
\text { Risk }\end{array}$ \\
\hline $\begin{array}{l}\text { Maldonado } \\
\text { (2009) }\end{array}$ & Low Risk & Uncertain & High Risk & Uncertain & Low Risk & Uncertain & $\begin{array}{l}\text { Low } \\
\text { Risk }\end{array}$ \\
\hline $\begin{array}{c}\text { Karaman } \\
(2015)\end{array}$ & Uncertain & Low Risk & High Risk & Uncertain & Low Risk & Uncertain & $\begin{array}{l}\text { Low } \\
\text { Risk }\end{array}$ \\
\hline $\begin{array}{l}\text { Djaiani } \\
(2016)\end{array}$ & Low Risk & Low Risk & High Risk & Low Risk & Low Risk & Low Risk & $\begin{array}{l}\text { Low } \\
\text { Risk }\end{array}$ \\
\hline $\begin{array}{l}\text { Liu a } \\
(2016)\end{array}$ & Low Risk & Uncertain & High Risk & Uncertain & Low Risk & Low Risk & $\begin{array}{l}\text { Low } \\
\text { Risk }\end{array}$ \\
\hline $\begin{array}{l}\text { Liu b } \\
(2016)\end{array}$ & Low Risk & Low Risk & High Risk & Low Risk & Low Risk & Low Risk & $\begin{array}{l}\text { Low } \\
\text { Risk }\end{array}$ \\
\hline $\begin{array}{l}\text { Mogahd } \\
(2017)\end{array}$ & Uncertain & Uncertain & High Risk & Uncertain & Low Risk & Uncertain & $\begin{array}{l}\text { Low } \\
\text { Risk }\end{array}$ \\
\hline $\begin{array}{c}\text { Elgebaly } \\
(2018)\end{array}$ & Uncertain & Uncertain & High Risk & Uncertain & Low Risk & Uncertain & $\begin{array}{l}\text { Low } \\
\text { Risk }\end{array}$ \\
\hline $\begin{array}{l}\text { Sheikh } \\
(2018)\end{array}$ & Low Risk & Low Risk & Low Risk & Low Risk & Low Risk & Uncertain & $\begin{array}{l}\text { Low } \\
\text { Risk }\end{array}$ \\
\hline Shi (2019) & Low Risk & Uncertain & High Risk & Uncertain & Low Risk & High Risk & $\begin{array}{l}\text { Low } \\
\text { Risk }\end{array}$ \\
\hline
\end{tabular}

The overall quality of the trials is moderate and all of them - except the study by Sheikh - lacked a proper blinding of participants and personnel.

\section{Figures}




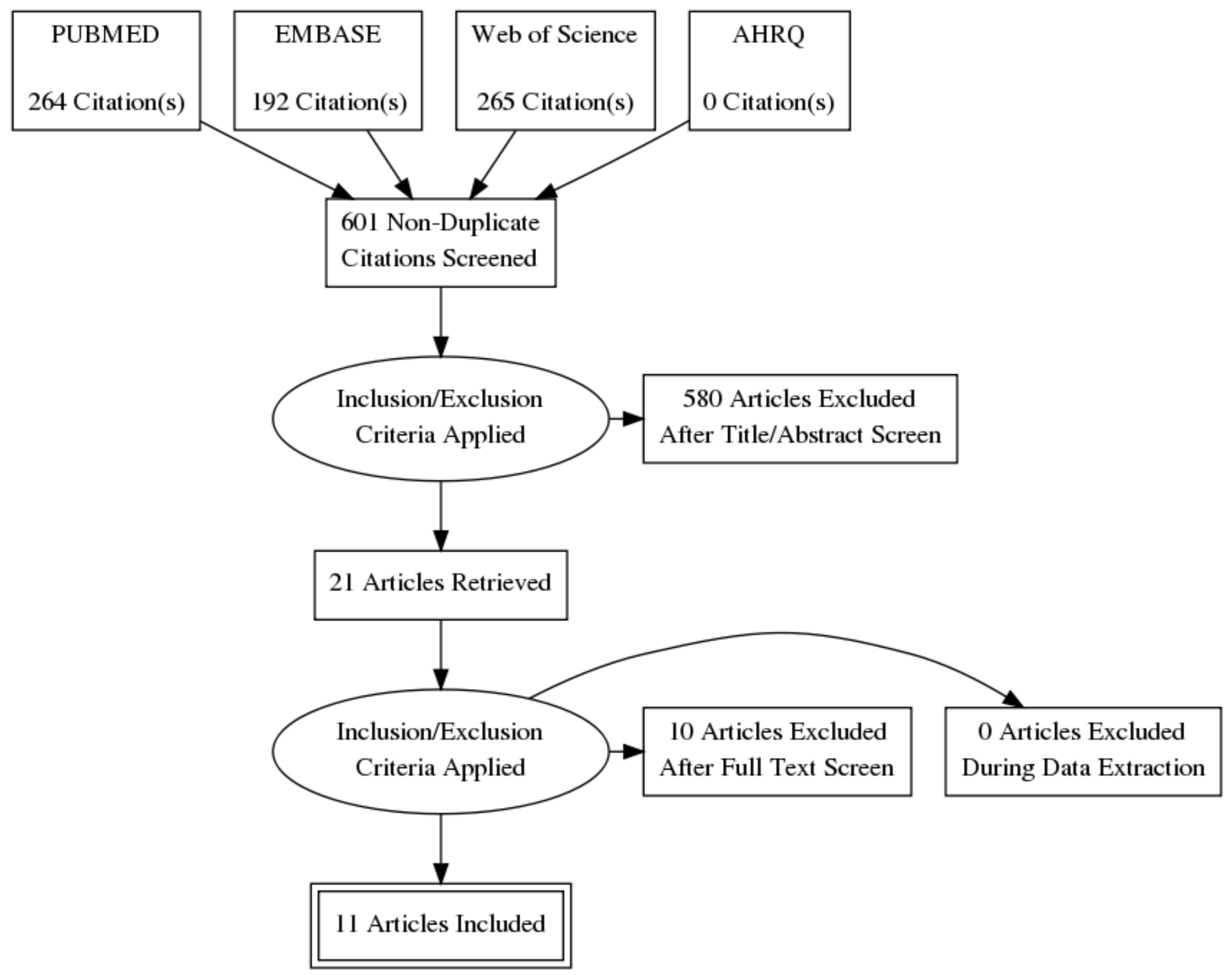

Figure 1

Study flow diagram. 
A

Study name

Statistics for each study

Std diff in means and $95 \% \mathrm{Cl}$

Std diff Standard Lower Upper

in means error Variance limit limit Z-Valuep-Value

$\begin{array}{llllllll}\text { Corbett } & -0.176 & 0.213 & 0.045 & -0.592 & 0.241 & -0.827 & 0.408\end{array}$

$\begin{array}{llllllll}\text { Maldonado } & -0.709 & 0.266 & 0.071 & -1.231 & -0.188 & -2.665 & 0.008\end{array}$

$\begin{array}{llllllll}\text { Djaiani } & -1.084 & 0.158 & 0.025 & -1.394 & -0.774 & -6.846 & 0.000\end{array}$

$\begin{array}{llllllll}\text { Liu a } & -0.560 & 0.217 & 0.047 & -0.986 & -0.134 & -2.578 & 0.010\end{array}$

$\begin{array}{llllllll}\text { Mogahd } & -0.370 & 0.241 & 0.058 & -0.843 & 0.102 & -1.535 & 0.125\end{array}$

$\begin{array}{llllllll}\text { Elgebaly } & -0.455 & 0.286 & 0.082 & -1.016 & 0.107 & -1.587 & 0.112\end{array}$

$\begin{array}{llllllll}\text { Sheikh } & -0.903 & 0.271 & 0.073 & -1.434 & -0.371 & -3.330 & 0.001\end{array}$

$\begin{array}{llllllll}\text { Shi } & -1.211 & 0.170 & 0.029 & -1.545 & -0.878 & -7.129 & 0.000\end{array}$

$\begin{array}{lllllllll}\text { Random } & -0.703 & 0.143 & 0.020 & -0.983 & -0.423 & -4.919 & 0.000\end{array}$

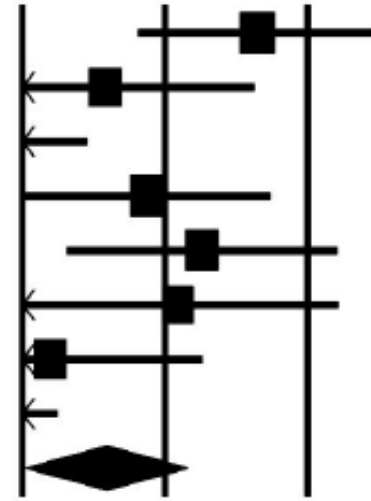

$\begin{array}{rrr}-1.00 & -0.50 & 0.00\end{array}$

Favours A

Dexmedetomidine

Favours B

Propofol

B

Study name

Statistics for each study

Std diff in means and $95 \% \mathrm{Cl}$

Std diff Standard

Lower Upper

in means error Variance limit limit Z-Valuep-Value

Herr

$-0.130$

0.117

$\begin{array}{llll}0.014 & -0.358 & 0.099 & -1.114\end{array}$

0.265

Corbett

0.117

0.212

$\begin{array}{llll}0.045 & -0.299 & 0.534\end{array}$

0.553

0.580

Mogahd

$-3.392$

0.373

$\begin{array}{llll}0.139 & -4.123 & -2.660 & -9.087\end{array}$

0.000

Elgebaly

0.192

0.283

$\begin{array}{llll}0.080 & -0.363 & 0.748 & 0.678\end{array}$

0.498

Sheikh

$-0.621$

0.264

$\begin{array}{lllll}0.070 & -1.139 & -0.102 & -2.348\end{array}$

0.019

Random

$-0.722$

0.446

$0.199-1.597$

$0.152-1.619$

0.105

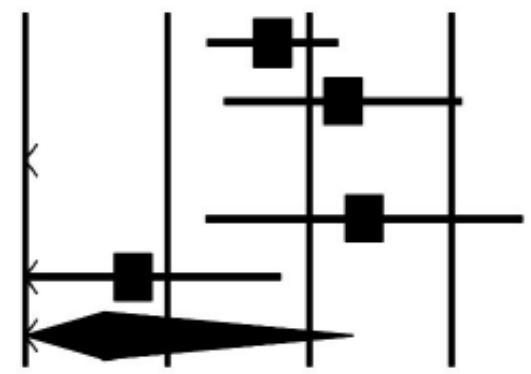

$-1.00$

$-0.50$

0.00

0.50

1.00
Favours B

Dexmedetomidine

Propofol

Figure 2

Time to Extubation and Mechanical Ventilation Time Standardized Mean Differences. (A): Time To Extubation; (B): Mechanical Ventilation Time. Heterogeneity was assessed using Cochran's Q test with $\mathrm{p}<0.05$ for statistical significance and $\mathrm{I} 2$ index $>50 \%$ for significant heterogeneity. 
Study name

Standard

Herr

Maldonado

Karaman

Djaiani

Liu a

Liu b

Mogahd

Shi

Random error Variancein means limit limit Z-Valuep-Value

$\begin{array}{llrrrrr}0.117 & 0.014 & -0.313 & -0.543 & -0.084 & -2.675 & 0.007 \\ 0.259 & 0.067 & 0.176 & -0.331 & 0.683 & 0.680 & 0.497 \\ 0.278 & 0.077 & -1.376 & -1.921 & -0.830 & -4.946 & 0.000 \\ 0.150 & 0.022 & -0.428 & -0.721 & -0.135 & -2.864 & 0.004 \\ 0.213 & 0.045 & -0.046 & -0.464 & 0.372 & -0.215 & 0.830 \\ 0.257 & 0.066 & -0.220 & -0.724 & 0.284 & -0.855 & 0.393 \\ 0.344 & 0.118 & -2.923 & -3.597 & -2.249 & -8.503 & 0.000 \\ 0.156 & 0.024 & -0.165 & -0.472 & 0.142 & -1.055 & 0.292 \\ 0.231 & 0.053 & -0.612 & -1.064 & -0.160 & -2.655 & 0.008\end{array}$

Statistics for each study

Std diff in means and $95 \% \mathrm{Cl}$

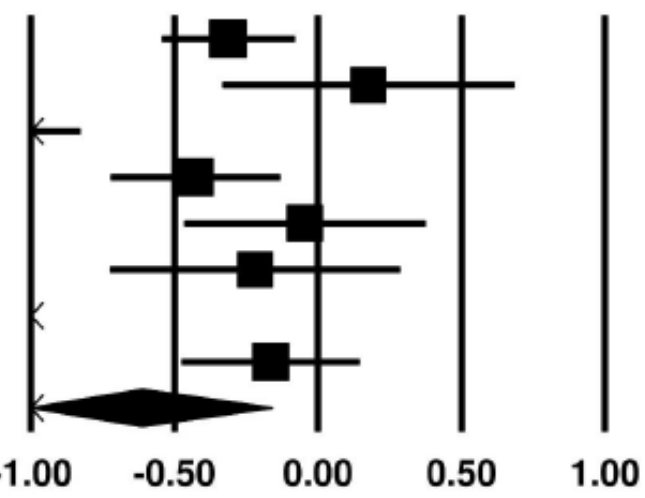

Favours A

Dexmedetomidine

Favours B

Propofol

\section{B}

Study name

Statistics for each study

Std diff in means and $95 \% \mathrm{Cl}$

\section{Std diff Standard Lower Upper \\ in means error Variance limit limit Z-Valuep-Value}

$\begin{array}{llllllll}\text { Maldonado } & -0.366 & 0.260 & 0.068 & -0.876 & 0.144 & -1.406 & 0.160 \\ \text { Djaiani } & -0.887 & 0.155 & 0.024 & -1.191 & -0.584 & -5.726 & 0.000 \\ \text { Liu a } & -0.016 & 0.213 & 0.045 & -0.433 & 0.402 & -0.073 & 0.942 \\ \text { Shi } & -3.269 & 0.239 & 0.057 & -3.737 & -2.801 & -13.694 & 0.000 \\ \text { Random } & -1.133 & 0.662 & 0.439 & -2.431 & 0.166 & -1.710 & 0.087\end{array}$

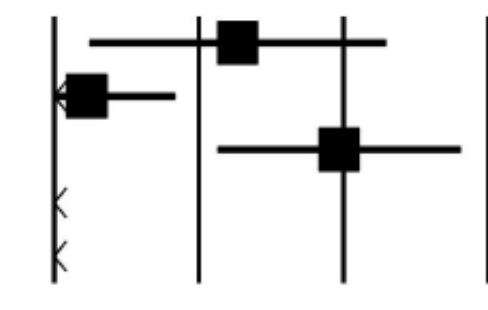

$-1.00$

$-0.50 \quad 0.00$

0.50

1.00

Favours A

Dexmedetomidine
Favours B Propofol

Figure 3

Length of Stay in the Intensive Care Unit and Length of Stay in the hospital Standardized Mean Differences. (A): Length Of Stay in ICU; (B): Length of Stay in the hospital. Heterogeneity was assessed using Cochran's $Q$ test with $p<0.05$ for statistical significance and 12 index $>50 \%$ for significant heterogeneity. 
Study name

\section{Odds ratio}

Maldonado

Djaiani

Liu a

Liu b

Sheikh

Shi

Random
0.034

0.463

0.081

0.207

0.113

1.818

0.683
Statistics for each study

Lower Upper

limit

0.004

0.231

0.004

0.010

0.013

0.937

0.437 limit

$0.287-3.116$

$\begin{array}{ll}0.930 & -2.165\end{array}$

$1.506-1.686$

$4.491 \quad-1.004$

$\begin{array}{ll}0.988 & -1.971\end{array}$

$\begin{array}{ll}3.528 & 1.767\end{array}$

$\begin{array}{ll}1.069 & -1.668\end{array}$
Odds ratio and $95 \% \mathrm{Cl}$

0.002
0.030
0.092
0.316
0.049
0.077
0.095

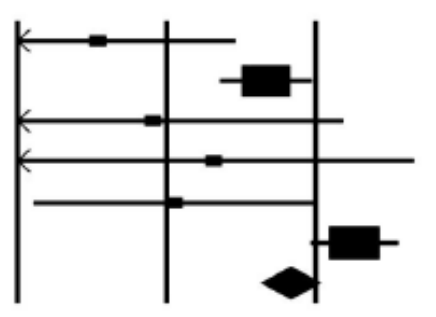

0.1

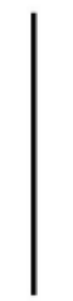

10 100

$\underset{\text { Dexmedetomidine }}{\text { Favours }}$ Propofol $B$

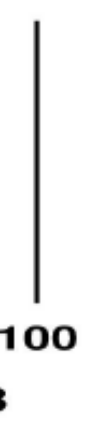

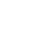

Study name

\section{Odds ratio}

Herr

Karaman

Liu a

Liu $b$

Fixed
2.535

2.207

4.300

6.458

3.397
Statistics for each study

Lower Upper limit limit Z-Value p-Value

$\begin{array}{llll}0.484 & 13.279 & 1.101 & 0.271\end{array}$

$\begin{array}{lll}0.190 & 25.638 & 0.633\end{array}$

$\begin{array}{lll}0.461 & 40.118 & 1.280\end{array}$

$0.707 \quad 59.001 \quad 1.653$

$\begin{array}{lll}1.208 & 9.552 & 2.319\end{array}$
0.527

0.200

0.098

0.020
Odds ratio and $95 \% \mathrm{CI}$

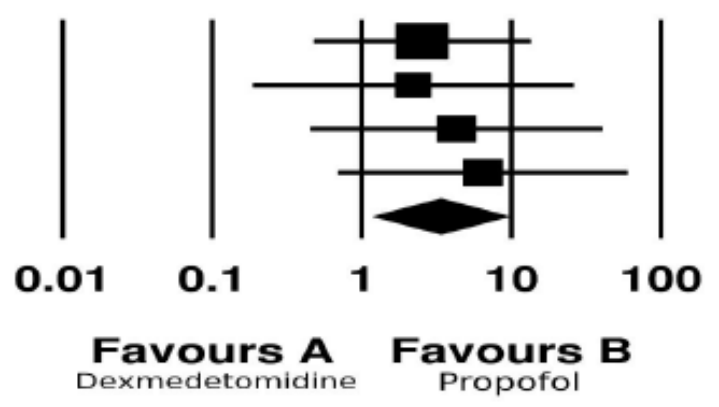

Odds ratio and $95 \% \mathrm{Cl}$

Statistics for each study

Odds Lower Upper

ratio limit limit Z-Value p-Value

Herr

Corbett

1.647

2.117

Karaman

0.926

0.791

2.932

1.697

5.668

1.493

0.830

Liu a

Liu b

3.138

0.201

3.422

$-0.258$

1.301

0.294

7.568

2.512

2.545

0.859

1.096

2.580

$-0.277$

2.380
0.090

0.136

0.796

0.011

0.781

0.017 $\mid$
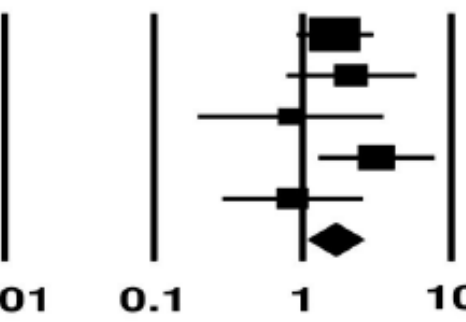

0.1

1 
This is a list of supplementary files associated with this preprint. Click to download.

- Supplementary1.pdf

- Supplementary2.pdf 